\title{
Panorama atual da doação de leite humano no Brasil: uma revisão integrativa
}

\author{
Current overview of the human milk donation in Brazil: an integrative revision
}

\author{
Andréa Bayma Pinheiro ${ }^{1}$, Aline Carolina Castro Mota², Anna Carolina Padilha Castro Gomes², \\ Yasmin Amorim dos Santos², Anderson Bentes de Lima3 ${ }^{3}$ Mauro de Souza Pantoja ${ }^{3}$
}

Resumo Objetivo: Identificar o panorama atual da Doação de Leite Humano no Brasil. Método: Revisão integrada de artigos originais e revisões que investigaram os fatores associados à Doação de Leite Humano no Brasil. Foram incluídos artigos originais e revisões, publicados e indexados no MedLine, SciELO e PubMed, publicados no período de 2014 a 2018. Resultados: Capacitações da equipe multiprofissional de Unidades Básicas de Saúde para a promoção da doação e captação de leite é eficaz. Incentivo à doação e orientação sobre a ordenha maximizam a doação de leite humano. A doação de leite coletado pelas mães em seus domicílios é eficaz, quando seguidas as normas de manipulação e armazenamento. Os Bancos de Leite Humano são ferramentas de desenvolvimento da área de saúde e de crescimento político-econômico do Brasil. Conclusão: Houve aumento da doação de leite humano ao longo dos anos, todavia essa prática ainda deve ser estimulada no Brasil.

Descritores: leite humano; doações; Brasil.

Abstract Purpose: The aim of this study is to identify the current panorama of the Human Milk Donation in Brazil. Method: An integrative revision of original research articles reporting the analyzes of the contributing factors associated with human milk donation in Brazil. Original articles and reviews, published and indexed in MedLine, SciELO and PubMed, published between 2014 and 2018. Results: Training of multi- professional teams in health basic units for the collection and promotion of human milk donation. The incentive of donation and orientations about milking maximize the action of human milk donation. The donation of expressed breast milk collected at home is effective provided persons follow the defined standards for manipulation and storage of human milk. Human milk banks are essential tools for the development of health services as well as have positive impact on the political and economic growth in Brazil. Conclusion: The donation of breast milk has been increasing over the years; however, this practice should be further stimulated in Brazil.

Keywords: human milk; gift giving; Brazil.

${ }^{1}$ Fundação Santa Casa de Misericórdia do Pará - FSCMP, Belém, PA, Brasil

${ }^{2}$ Universidade Federal do Pará - UFPA, Belém, PA, Brasil

3Universidade do Estado do Pará - UEPA, Belém, PA, Brasil

Fonte de financiamento: Nenhuma.

Conflito de interesses: Os autores declaram não haver conflitos de interesse.

Recebido: Maio 15, 2018

Aceito: Junho 26, 2018

Trabalho realizado na Fundação Santa Casa de Misericórdia do Pará - FSCMP, Belém, PA, Brasil. 


\section{Introdução}

O leite humano proporciona benefícios nutricionais, imunológicos, psicológicos e econômicos reconhecidos e inquestionáveis ${ }^{1,2}$. Estudos mostram que o bebê que for alimentado exclusivamente por leite materno durante os primeiros seis meses, terá uma possibilidade maior de uma vida saudável nesse período, devido ao fato do leite materno conter valores nutricionais relevantes, capazes de prevenir doenças infecciosas, anemia, alergias, pneumonias, além de favorecer um melhor desenvolvimento cognitivo e visual aos prematuros ${ }^{3}$. Para recém-nascidos pré-termo, essas qualidades adquirem relevo especial por sua maior vulnerabilidade ${ }^{1}$.

Em virtude disso, os Bancos de Leite Humano (BLHs) foram criados para garantir a qualidade do leite humano destinado a crianças recém nascidas prematuras, de baixo peso ou hospitalizadas em Unidades de Tratamento Intensivo Neonatal. Além disso, os BLHs incentivam o aleitamento materno, fornecem orientações sobre "pega" e posição durante a mamada e dão apoio e acompanhamento às puérperas e lactantes com dificuldade na prática da amamentação, atuando com medidas educativas para contribuir com a redução da mortalidade neonatal ${ }^{4}$. Esses centros realizam a coleta, o processamento e o controle do leite humano ordenhado, para, depois, distribuí-lo aos lactentes ${ }^{5}$, considerando as especificidades do recém nascido, sendo assim, é uma estratégia que permite que as mães participem ativamente no processo de lactação favorecendo, portanto, a continuidade do aleitamento materno ${ }^{6}$.

A prática da doação de leite humano ganhou notoriedade com o surgimento dos $\mathrm{BLHs}^{7}$, a qual é definida como uma ação realizada por nutrizes saudáveis que apresentam excesso da produção de leite, além das necessidades normais do lactente, e que se disponibilizam a doá-lo ${ }^{8}$. O processo de tomada de decisão para a doação pode ser influenciado pelo tipo de atendimento recebido, se é humanizado ou não, e pela valorização da autonomia, cabendo ressaltar que a comunicação adequada tem efeito fundamental nesse processo'.

\section{Método}

Optou-se pela realização de uma revisão integrada de artigos originais e revisões que investigaram os fatores associados à Doação de Leite Humano no Brasil. Este tipo de revisão agrupou e sumarizou resultados da pesquisa sobre o tema em foco, permitindo indicar falhas no conhecimento e realizar um resumo vários estudos publicados.

Durante a elaboração deste estudo, estabeleceram-se 8 etapas: estabelecimento da questão da pesquisa; seleção dos descritores; escolha das bases de dados; definição dos critérios de inclusão e exclusão; categorização; avaliação dos estudos e interpretação dos resultados.

Como estratégia de busca, foram utilizados os seguintes descritores (Descritores em Ciência da SaúdeDeCS): Doação de leite; Banco de leite; Brasil. Foram executados a cooptação de dois descritores por vez e aplicado os filtros: "Doação" de Leite" + "Brasil"; "Banco de Leite" + "Brasil". Ainda foi aproveitada a tática de busca nas referências bibliográficas dos artigos selecionados, com objetivo de identificar os que porventura não tivessem sido encontrados na busca inicial. As bases de dados escolhidos para a pesquisa foram: MEDLINE, SciELO e PubMed, publicados no período de 2014 até 2018. A busca foi realizada em Fevereiro de 2018.

Foram encontradas 49 publicações e, como critérios de inclusão, foram selecionados artigos provenientes de pesquisas originais e revisões, nos idiomas inglês e português, e que contemplasse o maior número de artigos relacionados à pesquisa em questão. Foram excluídos da análise os relatos de caso, estudos que não contemplavam o objetivo da pesquisa e as produções realizadas em outros país e que não estavam nas línguas selecionadas.

Este estudo de revisão foi composto de 6 artigos, de acordo com os parâmetros de busca mencionado. Após a seleção dos artigos, foi elaborado um quadro para reunir e sintetizar as informações a serem extraídas dos textos, contendo as seguintes questões: título; ano de publicação; objetivo do estudo; metodologia do estudo e principais resultados. Essa sumarização permitiu uma síntese do conhecimento observado na literatura, dividida de acordo com os fatores associados à Doação de Leite Humano no Brasil, conforme mostra a Tabela 1. 
Tabela 1. Sumarização dos artigos que constituem a amostra da revisão integrativa

\begin{tabular}{|c|c|c|c|}
\hline Artigo / Ano & Objetivo & Métodos & Resultados \\
\hline $\begin{array}{l}\text { “Educação Popular } \\
\text { em Saúde: doação } \\
\text { de leite humano em } \\
\text { comunidade do Rio } \\
\text { de Janeiro, Brasil." } \\
\text { Pellegrine et al, 2014. }\end{array}$ & $\begin{array}{l}\text { Relatar a experiência } \\
\text { acerca da promoção do } \\
\text { aleitamento materno com } \\
\text { nutrizes para a doação } \\
\text { de leite humano em uma } \\
\text { UBS de uma comunidade } \\
\text { do município do Rio de } \\
\text { Janeiro. }\end{array}$ & $\begin{array}{l}\text { Relato de experiência } \\
\text { desenvolvida no período de } 2011 \\
\text { a } 2013 \text { em uma UBS. Foi dividida } \\
\text { em etapas: capacitação dos } \\
\text { profissionais da UBS; captação } \\
\text { e seleção das doadoras; grupos } \\
\text { educativos desenvolvidos com } \\
\text { as nutrizes; orientações para } \\
\text { ordenha; coleta, conservação e } \\
\text { transporte de leite materno para } \\
\text { os BLHs. Essa ação foi aplicada } \\
\text { por profissionais da UBS, tais } \\
\text { quais agentes comunitários de } \\
\text { saúde, enfermeiros, técnicos de } \\
\text { enfermagem, dentistas, auxiliares } \\
\text { de higiene dental, técnicos } \\
\text { administrativos e médicos. }\end{array}$ & $\begin{array}{l}\text { A captação e doação de leite } \\
\text { materno em USB mostrou-se uma } \\
\text { eficaz ampliação do trabalho } \\
\text { realizado pelos BLHs, pois } \\
\text { acompanha e orienta no próprio } \\
\text { domicílio da nutriz o manejo } \\
\text { da amamentação, identificando } \\
\text { precocemente possíveis } \\
\text { situações de risco ao aleitamento } \\
\text { materno. Dessa forma, essa } \\
\text { experiência favoreceu a promoção } \\
\text { ao aleitamento materno, } \\
\text { proporcionou maior confiança } \\
\text { no ato da doação de leite para } \\
\text { os BLHs, sendo percebido um } \\
\text { aumento de doadoras de leite } \\
\text { humano e da produção de leite } \\
\text { humano doado. }\end{array}$ \\
\hline
\end{tabular}

Estudo transversal desenvolvido em 2009 no Banco de leite do Hospital das Clínicas da Universidade Federal de Uberlândia (BLH HC/UFU). Participaram do estudo 48 mulheres com idade igual ou superior a 18 anos da região urbana de Uberlândia, as quais concordaram em participar do estudo. Foram realizadas visitas domiciliares para observar os procedimentos realizados durante a extração e armazenamento de leite humano, sendo as observações anotadas de acordo com a lista de verificação das técnicas do banco de leite.
"Prevalência e fatores associados à doação de leite para postos de recebimento de leite humano de unidades básicas de saúde." Meneses, Oliveira e Boccolini, 2016.
Estimar a prevalência e analisar os fatores associados à doação de leite materno em unidades básicas de saúde com vistas a aumentar os estoques dos bancos de leite humano.
Estudo transversal conduzido em 2013 mediante entrevista de 695 mães de crianças menores de um ano assistidas nas nove unidades básicas de saúde com posto de recebimento de leite humano ordenhado.

\author{
“O diálogo entre \\ saúde e política \\ externa na \\ cooperação brasileira \\ em bancos de leite \\ humano." Pittas, Dri \\ 2017
}

Compreender o que motiva o Brasil a promover os bancos de leite internacionalmente.
O artigo faz uma análise teórica baseada no conceito de soft power e está dividido em três seções: apresentação do referencial teórico, que consiste no soft power de Nye; apresentação do contexto em que se encontra o modelo brasileiro em BLH; por fim, interpretação da estratégia e dos resultados brasileiros.
Apenas 7,3\% das 695 mães haviam doado leite materno às UBSs. Os fatores que aumentaram a prevalência da doação de leite foram o incentivo à doação de leite por profissionais de saúde, parentes ou amigos, a orientação da unidade básica sobre a ordenha das mamas e ajuda na amamentação oferecida pela unidade. Porém, a internação do bebê em unidade neonatal associou-se a uma menor prevalência na doação de leite.

O estudo mostra que os projetos internacionais brasileiros em bancos de leite humano são eficazes para estabelecer contatos internacionais, mesmo sendo uma tecnologia de baixo custo. Além disso, conferem ao país autonomia e demonstram capacidade de liderança e geram prestígio. 
Tabela 1. Continuação...

\begin{tabular}{|c|c|c|c|}
\hline Artigo / Ano & Objetivo & Métodos & Resultados \\
\hline $\begin{array}{l}\text { "Práticas educativas } \\
\text { segundo os “Dez } \\
\text { passos para o sucesso } \\
\text { do aleitamento } \\
\text { materno" em um } \\
\text { Banco de Leite } \\
\text { Humano." Silva et } \\
\text { al, } 2017\end{array}$ & $\begin{array}{l}\text { Avaliar práticas } \\
\text { educativas segundo } \\
\text { os “Dez Passos para o } \\
\text { Sucesso do Aleitamento } \\
\text { Materno" em um Banco } \\
\text { de Leite Humano, a fim } \\
\text { de aumentar a adesão à } \\
\text { prática do aleitamento. }\end{array}$ & $\begin{array}{l}\text { Estudo retrospectivo com dados } \\
\text { secundários obtidos a partir de } \\
\text { formulário de preenchimento } \\
\text { obrigatório de } 12.283 \text { mães } \\
\text { atendidas em um Banco de Leite } \\
\text { Humano de uma maternidade em } \\
\text { Belo Horizonte (BH), com o título } \\
\text { de Hospital Amigo da Criança. }\end{array}$ & $\begin{array}{l}\text { Os achados apontaram para a } \\
\text { importância do profissional da } \\
\text { saúde no treinamento mãe/ } \\
\text { filho sobre aleitamento materno } \\
\text { e incentivo ao contato pele/pele, } \\
\text { amamentação exclusiva e sob } \\
\text { livre demanda, sendo necessário } \\
\text { aprimorar as orientações ofertadas } \\
\text { a fim de reduzir o uso de bicos } \\
\text { artificiais e potencializar a } \\
\text { amamentação exclusiva. }\end{array}$ \\
\hline $\begin{array}{l}\text { “Quality of human } \\
\text { milk expressed in a } \\
\text { human milk bank } \\
\text { and at home." Borges } \\
\text { et al, } 2017\end{array}$ & $\begin{array}{l}\text { Avaliar a qualidade do leite } \\
\text { humano ordenhado em } \\
\text { domicílio e no Banco de } \\
\text { Leite Humano. }\end{array}$ & $\begin{array}{l}\text { Estudo retrospectivo, realizado por } \\
\text { meio da avaliação dos registros da } \\
\text { acidez titulável e dos resultados } \\
\text { de cultura microbiológica de } \\
100 \text { amostras de leite humano } \\
\text { ordenhado em domicílio e em um } \\
\text { Banco de Leite Humano, no ano } \\
\text { de } 2014 \text {. }\end{array}$ & $\begin{array}{l}\text { Não foi detectada diferença } \\
\text { significativa da acidez e coleção } \\
\text { microbiológica entre os dois } \\
\text { grupos de amostras, sendo } \\
\text { que } 98 \% \text { e } 94 \% \text { das amostras } \\
\text { foram aprovadas entre as } \\
\text { coletadas no BLH e em domicílio } \\
\text { respectivamente. }\end{array}$ \\
\hline
\end{tabular}

\section{Resultados}

A partir da análise da literatura sobre a doação de leite humano no Brasil e a participação dos BLHs, percebe-se que a literatura ainda é escassa em relação ao tema, mas que tem aumentado, principalmente no ano de 2017, no qual foi encontrado o maior número de artigos (Tabela 1). Além disso, a maior parte dos estudos ratifica a importância de promover doação, não só nos hospitais, mas também nos domicílios das mães.

A realização das capacitações da equipe multiprofissional de Unidades Básicas de Saúde para a promoção da doação e captação de leite mostrou-se eficaz, pois aumentou a confiança das mães em realizar a contribuição, aumentando o número de doadoras e a quantidade de leite produzido nos $\mathrm{BLHs}^{7}$. O incentivo a doação, tal como a orientação sobre a ordenha, são importantes fatores que maximizam a oferta de leite humano ${ }^{10}$ e a adoção dos "Dez Passos para o Sucesso do Aleitamento Materno" é uma ferramenta fundamental para o crescimento de mulheres doadoras de leite ${ }^{11}$.

Ademais, a doação de leite coletado pelas mães em seus domicílios é eficaz, quando seguidas as técnicas e normas de manipulação e armazenamento do leite, da mesma forma que o leite coletado nos BLHs ${ }^{12,13}$, mostrando que essa é uma pratica que deve ser fomentada no Brasil.

Nota-se ainda que os BLHs são ferramentas não só de desenvolvimento da área de saúde do Brasil, mas também de crescimento político-econômico ${ }^{14}$. Logo, nos artigos estudados, os BLHs mostraram-se essenciais na atual realidade brasileira.

Desse modo, é possível verificar, de acordo com esses artigos, os principais fatores que estão envolvidos com a doação de leite humano, podendo ser divididos em fatores humanos, locacionais e operacionais (Figura 1).

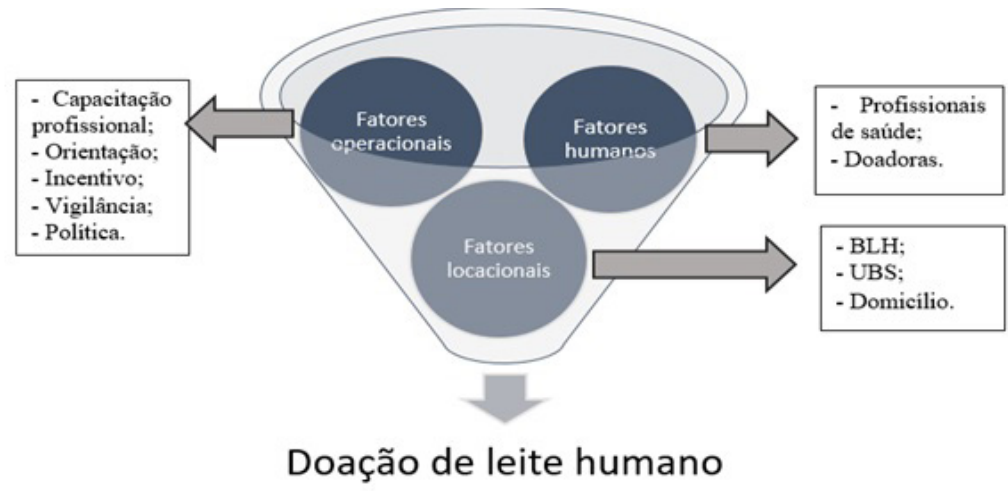

Figura 1. Fatores envolvidos da doação de leite. 


\section{Discussão}

A Política Nacional de Aleitamento Materno está pautada, principalmente, na elaboração e realização de seis estratégias, as quais incluem ações na atenção básica e hospitalar, criação da Rede Brasileira de Bancos de Leite Humano (BLH), proteção legal ao aleitamento materno, divulgação de campanhas na mídia e monitoramento dos indicadores de aleitamento materno ${ }^{15}$.

Paralelamente a isso, há os BLHs, os quais são unidades especializadas e vinculadas à unidade de terapia neonatal e uma de suas missões diz respeito à estimulação da doação de leite materno. São também responsáveis por ações de promoção, proteção e apoio ao aleitamento materno e execução de atividades de coleta da produção lática da nutriz, seleção, classificação, processamento, controle de qualidade e distribuição.

Tendo como base estudos acerca da Doação de Leite humano, observa-se que, por vezes, tal ato ainda é incipiente, visto que resultados encontrados em estudo realizado no município do Rio de Janeiro mostram que das 695 mães de crianças menores de um ano nas nove unidades com Posto de Recebimento de Leite Humano Ordenhado apenas 7,3\% destas haviam doado leite materno às unidades básicas de saúde ${ }^{11}$. Essa realidade de doação vem, paulatinamente, se modificando ao longo dos anos visto que as questões de saúde têm ganhado força em função de aspectos como a globalização.

Tendo em vista que aspectos como a globalização e a intensificação nas interações humanas geraram maior interconectividade espacial e temporal, têm-se, atualmente, a tendência brasileira de se produzir projetos internacionais brasileiros em BLHs ${ }^{7}$. Inicialmente, foi exposta a relevância das políticas em BLHs tanto em termos globais como nacionalmente. O modelo brasileiro dispõe de mecanismos que lhe conferem grande eficácia, mesmo sendo uma tecnologia de baixo custo. Em consequência, torna-se o modelo ideal para países em desenvolvimento ${ }^{14}$.

Além disso, também é notória a necessidade de capacitação atrelada à doação, de modo a viabilizar o ato de maneira mais segura e eficaz. Mediante esse entendimento, um estudo realizado em uma Unidade Básica de Saúde (UBS), localizada em uma comunidade do município do Rio de Janeiro, promoveu projeto de Educação Popular em Saúde acerca da promoção do aleitamento materno com nutrizes para a doação de leite humano ${ }^{7}$. A atuação ocorreu por meio de grupos educativos que promoviam orientações individuais na Unidade e nas visitas domiciliares, agindo sobre a realidade de cada nutriz, de modo a aumentar a finalidade. Dessa maneira, observa-se que o contexto no qual as nutrizes foram inseridas propicia um maior entendimento da necessidade de doação, além de proporcionar maior confiança no ato da doação de leite 7 .

Ainda sobre a necessidade de capacitação do ato de extração de leite humano para doação, um estudo realizado na cidade Uberlândia, em Minas Gerais, avaliou os procedimentos recomendados para extração domiciliar e transporte de leite humano, onde observou-se a execução correta da extração em um número considerável das doadoras, visto que $93,8 \%$ delas utilizavam proteção para os cabelos, cerca de $84 \%$ realizavam a lavagem das mãos antes da extração, além de se observar que $87,5 \%$ das doadoras apresentavam cuidados com as unhas e que outras 33,3\% desempenhavam o desprezo dos primeiros jatos ${ }^{13}$.

O desempenho de tais medidas possibilita uma extração mais segura e afastada de possíveis agentes microbianos advindos, das unhas, dos cabelos e das mãos. Dessa forma, um estudo realizado no Hospital de Clínicas de Uberlândia, em Minas Gerais ${ }^{12}$, busca avaliar a qualidade do leite ordenhado em domicílio e nos BLHs, por meio de registros de sua acidez e de aspectos microbiológicos, sendo que no estudo não foram detectadas diferenças significativas, o que pode indicar uma concordância com as práticas indicadas para a extração, transporte e armazenamento do leite humano ${ }^{12}$.

Inúmeras são as dificuldades encontradas pelas puérperas na prática da amamentação, dentre elas destacam-se: falhas no processo de orientação e falta de incentivo à amamentação desde o prénatal. Tais fatores isolados ou associados fazem com que as lactantes não amamentem seus filhos até o mínimo de tempo de seis meses proposto pela Organização Mundial da Saúde (OMS) ${ }^{16}$.

Ademais, paralelo às práticas citadas para uma boa extração, segura e eficaz, outro estudo realizado em um BLH de uma maternidade em Belo Horizonte $(\mathrm{BH})$ aponta para a importância do profissional da saúde no treinamento mãe/filho sobre aleitamento materno, bem como incentivo ao contato entre ambos, sendo observado ainda a necessário de se reduzir o uso de bicos artificiais, afim de fomentar a amamentação exclusiva ${ }^{11}$.

Pode-se citar também como uma das dificuldades no manejo clínico do recém-nascido a relação com dieta, principalmente devido à imaturidade gastrointestinal. O leite humano é mais tolerado pois apresenta nutrientes adequados, que estimulam o amadurecimento da defesa e do trofismo do trato gastrointestinal. Quanto mais precoce for a introdução do leite humano, maior é a chance de sobrevida e recuperação do recém-nascido ${ }^{17}$. 


\section{Conclusão}

Apesar da literatura sobre o tema ser escassa, é perceptível o aumento da doação de leite humano ao longo dos anos e tal aumento está relacionado a fatores como a globalização. A grande maioria dos estudos enfatiza a importância de promover a doação, não só nos hospitais, mas também nos domicílios das contribuintes. A capacitação das equipes multiprofissionais de saúde são de extrema importância para essa promoção.

Estudos realizados no Brasil comprovaram que a doação de leite coletado pelas lactantes em seus domicílios é eficaz, desde que as técnicas e normas de manipulação e armazenamento do leite sejam seguidas da mesma forma que o leite coletado nos BLHs.

A doação de leite humano deve estar atrelada a medidas de boa extração, transporte e armazenamento do leite, de modo a propiciar sua correta utilização. Essa prática deve ser estimulada no Brasil pois a política brasileira dos BLHs dispõe de mecanismos que lhe conferem grande eficácia, apesar de ser uma tecnologia de baixo custo. Desse modo, os BLHs são essenciais na atual realidade brasileira, de modo que promovem o desenvolvimento da Saúde e o crescimento político e econômico.

\section{Referências}

1. Nascimento MBR, Issler H. Aleitamento materno em prematuros: manejo clínico hospitalar. J Pediatr. 2004;80(5 Supl):163-72. http://dx.doi. org/10.2223/1250.

2. Algarves TR, Julião AMS, Costa HM. Aleitamento materno: influência de mitos e crenças no desmame precoce. Rev Saúde Foco. 2015;2(1):151-67.

3. Oliveira AS, Silva RCR, Fiaccone RL, Pinto EJ, Assis AM. Efeito da duração da amamentação exclusiva e mista sobre os níveis de hemoglobina nos primeiros seis meses de vida: um estudo de seguimento. Cad Saude Publica. 2010;26(2):409-17. http://dx.doi.org/10.1590/S0102311X2010000200020. PMid:20396856.

4. Maia PRS, Almeida JAG, Novak FR, Silva DA. Rede nacional de bancos de leite humano: gênese e evolução. Rev Bras Saude Mater Infant. 2006;6(3):285-92. http://dx.doi.org/10.1590/S1519-38292006000300004.

5. Almeida JAG, Guimarães V, Novak FR. BLH-IFF/NT- 16.04 - Ordenha: procedimentos higiênico sanitários. In: Rede Nacional de Bancos de Leite Humano. Normas técnicas para bancos de leite humano [Internet]. Rio de Janeiro: Centro de Referência Nacional para Bancos de Leite Humano, Instituto Fernandes Figueira, Fundação Oswaldo Cruz, Ministério da Saúde; 2004 [citado em 2018 out 08]. p. 1-7. Disponível em: http://www.redeblh.fiocruz.br/media/coleta.pdf

6. Conceição CS, Alves VH, Silva LR, Martins CA, Mattos DV, Rodrigues DP. Qualidade assistencial do Banco de Leite Humano: percepção de usuárias. Rev Enferm UFPE. 2013;7(5):1271-78. https://doi.org/10.5205/1981-8963-v7i5a11609p1271-1278-2013.

7. Pellegrine JB, Koopmans FF, Pessanha HL, Rufino CG, Farias HPS. Educação popular em saúde: doação de leite humano em comunidade do Rio de Janeiro, Brasil. Interface Comunicacao Saude Educ. 2015;18(Supl 2):1499-506. http://dx.doi.org/10.1590/1807-57622013.0496.

8. Brasil. Banco de leite humano: funcionamento, prevenção e controle de riscos. Brasília: Ministério da Saúde; 2008.

9. Normas técnicas para bancos de leite humano. Ordenha: procedimentos higiênicosanitários. 2004. [acesso em: 08 outubro 2006]. Disponível em: http://www.redeblh.fiocruz.br/media/coleta.pdf

10. Meneses TMX, Oliveira MIC, Boccolini CS. Prevalence and factors associated with breast milk donation in banks that receive human milk in primary health care units. J Pediatr. 2017;93(4):382-8. http://dx.doi.org/10.1016/j.jped.2016.09.004. PMid:28432863.

11. Silva CME, Pellegrinelli ALR, Pereira SCL, Passos IR, Santos LCD. Práticas educativas segundo os “Dez passos para o sucesso do aleitamento materno" em um Banco de Leite Humano. Rev Cien Saude Colet. 2017;11(1):1661-71. http://dx.doi.org/10.1590/1413-81232017225.14442015.

12. Borges MS, Oliveira AMM, Hattori WT, Abdallah VOS. Quality of human milk expressed in a human milk bank and at home. J Pediatr. 2018;94(4):399-403. PMid:28859913.

13. Menezes G, Lima-Cavalcanti L, Morais-Oliveira AM, Melo Costa-Pinto R, Steffen-Abdallah VO. Evaluación de la recolección domiciliaria realizada por un banco de leche humana de un hospital universitario de Brasil. Salud Publica Mex. 2014;56(3):245-50. http://dx.doi. org/10.21149/spm.v56i3.7341. PMid:25272175.

14. Pittas TM, Dri CF. O diálogo entre saúde e política externa na cooperação brasileira em bancos de leite humano. Cien Saude Colet. 2017;22(7):2277-86. http://dx.doi.org/10.1590/1413-81232017227.02832017. PMid:28724009.

15. Ministério da saúde. Políticas públicas de incentivo ao aleitamento materno: a experiência do Brasil [Internet]. Brasília: Ministério da Saúde; 2010 [citado em 2018 fev 21]. Disponível em: http://portal.saude.gov.br/portal/arquivos/pdf/artigo_franca_novo.pdf

16. Mascarenhas ACL, Miranda LTT, Brasil G B, Moia LJMP, Pimental IM, Lima VLA. A percepção das puérperas frenteà atuação do enfermeiro na promoção do aleitamento materno em um hospital amigo da criança no Estado do Pará. Revista Paraense de Medicina. 2015;29(3):1-11.

17. Maciel IVL, Almeida CS, Braga PP. Breastfeeding in the context of prematurity: the maternal speech. Rev Enferm UFPE. 2014;8(5):1-11. 


\section{Autor correspondente}

Andréa Bayma Pinheiro

Fundação Santa Casa de Misericórdia do Pará - FSCMP

Rua Oliveira Belo, 395, Umarizal, Belém, PA, Brasil

E-mail: andrea.bayma401@gmail.com

Informação sobre os autores

ABP é médica Pediatra especialista em Neonatologia.

ACCM, ACPCG e YAS são acadêmicas de Medicina.

$A B L$ é doutor em Biotecnologia.

MSP é doutor em Cirurgia.

Contribuição dos autores

ABP guiou a pesquisa e aperfeiçoou o artigo; $A C C$ fez a revisão bibliográfica e escreveu o artigo;

ACPCG fez a revisão bibliográfica e escreveu o artigo; YAS fez a revisão bibliográfica e escreveu o artigo; ABL realizou a revisão final do artigo; MSP orientou a pesquisa. 